\title{
Validation of Analytical Methods
}

\author{
Tentu Nageswara Rao \\ Additional information is available at the end of the chapter
}

http://dx.doi.org/10.5772/intechopen.72087

\begin{abstract}
Method validation is a key element in the establishment of reference methods and within the assessment of a laboratory's competence in generating dependable analytical records. Validation has been placed within the context of the procedure, generating chemical data. Analytical method validation, thinking about the maximum relevant processes for checking the best parameters of analytical methods, using numerous relevant overall performance indicators inclusive of selectivity, specificity, accuracy, precision, linearity, range, limit of detection (LOD), limit of quantification (LOQ), ruggedness, and robustness are severely discussed in an effort to prevent their misguided utilization and ensure scientific correctness and consistency among publications.
\end{abstract}

Keywords: method validation, accuracy, precision, linearity, LOD, LOQ

\section{Introduction}

Analytical method validation is an essential requirement to perform the chemical evaluation [1-3]. Method validation is a procedure of performing numerous assessments designed to verify that an analytical test system is suitable for its intended reason and is capable of providing beneficial and legitimate analytical data [4-8]. A validation examine includes testing multiple attributes of a method to determine that it may provide useful and valid facts whilst used robotically [9-11]. To accurately investigate method parameters, the validation test ought to consist of normal test conditions, which includes product excipients [11-14]. Therefore, a method validation examine is product-specific.

\section{Procedure}

\subsection{Parameters to be checked for method validation}

- $\quad$ Selectivity/Specificity

- Precision

\section{IntechOpen}

(c) 2018 The Author(s). Licensee IntechOpen. This chapter is distributed under the terms of the Creative Commons Attribution License (http://creativecommons.org/licenses/by/3.0), which permits unrestricted use, distribution, and reproduction in any medium, provided the original work is properly cited. (cc) BY 
- Accuracy

- $\quad$ Linearity

- Range

- Stability

- $\quad$ Limit of Detection (LOD) and Limit of Quantitation (LOQ)

\subsubsection{Selectivity/specificity}

Selectivity of an analytical method is its ability to measure accurately an analyte in the presence of interferences that may be expected to be present in the sample matrix.

Selectivity is checked by examining chromatographic blanks (from a sample that is known to contain no analyte) in the expected time window of the analyte peak. And the raw data for selectivity will be recorded in the raw data in approved formats.

\subsubsection{Precision}

Precision of a method is the degree of agreement among individual test results when the procedure is applied repeatedly to multiple samplings.

Precision is measured by injecting a series of standards or analyzing series of samples from multiple samplings from a homogeneous lot. From the measured standard deviation (SD) and Mean values, precision as relative standard deviation (\% rsd) is calculated.

$$
\% \text { rsd or CV }=\frac{\mathrm{SD}}{\text { Mean }} \times 100
$$

The raw data for precision will be recorded in the approved format and the acceptance criteria for precision will be given in the respective study plan or amendment to the study plan.

\section{OR}

Precision can be also calculated by using Horwitz equation:

The acceptable percent of relative standard deviation results for precision may be based on the Horwitz equation, an exponential relationship between the among-laboratory relative standard deviation $\left(\mathrm{RSD}_{\mathrm{R}}\right)$ and Concentration $(\mathrm{C})$ : [15]

$$
\% \mathrm{RSD}_{\mathrm{R}}=2^{(1-0.5 \log \mathrm{C})}
$$

For estimation of repeatability (RSDr), is modified to:

$$
\% \mathrm{RSD}_{\mathrm{r}}=\% \mathrm{RSD}_{\mathrm{R}} \times 0.67
$$

The Horwitz curve has been empirically derived and has been proven to be more or less independent of analyte, matrix and method of evaluation over the concentration range $\mathrm{C}=1$ (100\%) to $C=10^{-9}$ by the evaluation of vast numbers of method precision studies. The 
modified Horwitz values for repeatability $\mathrm{CV}$ given under may be used for guidance. If measured repeatability is outside those values, suggested explanation must be submitted for consideration. The details were presented in Table 1.

\subsubsection{Accuracy}

The accuracy of an analytical method is the degree of agreement of test results generated by the method to the true value.

Accuracy is measured by spiking the sample matrix of interest with a known concentration of analyte standard and analyzing the sample using the "method being validated." The procedure and calculation for Accuracy (as\% recovery) will be varied from matrix to matrix and it will be given in respective study plan or amendment to the study plan.

\subsubsection{Linearity}

The linearity of an analytical method is its capability to elicit check consequences which might be at once, or with the aid of well described mathematical adjustments, proportional to the concentration of analytes in within a given range.

Linearity is determined by injecting a series of standards of stock solution/diluted stock solution using the solvent/mobile phase, at a minimum of five different concentrations in the range of $50-150 \%$ of the expected working range. The linearity graph will be plotted manually/ using Microsoft Excel or software of the computer (Concentration vs. Peak Area Response) and which will be attached to respective study files.

\subsubsection{Range}

The range of an analytical method is the interval between the upper and lower levels that have been demonstrated to be determined with precision, accuracy and linearity using the set method. This range will be the concentration range in which the Linearity test is done.

\begin{tabular}{ll}
\hline Percent of analyte & $\begin{array}{l}\text { Proposed acceptable } \% \text { RSD }_{\mathbf{r}} \\
\text { (Horwitz value } \times \mathbf{0 . 6 7 )}\end{array}$ \\
\hline 100.00 & 1.340 \\
50.00 & 1.490 \\
20.00 & 1.710 \\
10.00 & 1.900 \\
5.00 & 2.100 \\
2.00 & 2.410 \\
1.00 & 2.680 \\
0.25 & 3.300 \\
\hline
\end{tabular}

Note: The unmodified Horwitz equation is used as a criterion of acceptability for methods collaboratively tested by CIPAC.

Table 1. Details of Horwitz values. 


\subsubsection{Stability}

Many analytes readily decompose prior to chromatography investigations, for example during the preparation of the sample solutions, during extraction, clean-up, phase transfer, and during storage of prepared vials. Under these circumstances, method development should investigate the stability of the analyte. Accuracy test takes care of stability. It is required to mention in the method how long a sample after extraction can be stored before final analysis, based on the duration taken for accuracy test.

\subsubsection{Limit of detection and limit of quantitation}

The term LOD is defined as the lowest concentration at which the instrument is able to detect but not quantify and the noise to signal ratio for LOD should be 1:3. The term LOQ is defined as the lowest concentration at which the instrument is able to detect and quantify. The noise to signal ratio for LOQ should be 1:10.

Determination of Limit of Detection (LOD) and Limit of Quantitation (LOQ) from Detector Linearity experiments (applicable to only instrument sensitivity).

LOD and LOQ values are calculated manually by taking Noise to signal ratio of a lowest/ known concentration of linearity samples and it will be expressed in $\mu \mathrm{g} / \mathrm{ml}$ or $\mathrm{ppm}$. To calculate in \%, values of LOD and LOQ will be multiplied by 100/lowest or known concentration of test item $(\mathrm{mg} / \mathrm{L})$ taken for analysis of that particular a.i. or impurity analysis.

Calculations of LOD and LOQ values for instrument sensitivity:

$$
\begin{aligned}
& \operatorname{LOD}(\mathrm{mg} / \mathrm{L})=3 \times \frac{\text { Noise }}{\text { Signal }} \times \text { Lowest concentration of the linearity samples } \\
& \mathrm{LOQ}(\mathrm{mg} / \mathrm{L})=10 \times \frac{\text { Noise }}{\text { Signal }} \times \text { Lowest concentration of the linearity samples }
\end{aligned}
$$

\section{Calculations of LOD and LOQ values for method:}

$$
\begin{aligned}
& \operatorname{LOD}(\%)=\frac{\operatorname{LOD}(\mathrm{mg} / \mathrm{L})}{\text { Test item conc.used for quantification }} \times 100 \\
& \operatorname{LOQ}(\%)=\frac{\mathrm{LOD}(\mathrm{mg} / \mathrm{L})}{\text { Test item conc.used for quantification }} \times 100
\end{aligned}
$$

\section{OR}

\subsubsection{Mathematical derivations}

\subsubsection{Determination of limit of detection (LOD) and limit of quantitation (LOQ)}

Prepare a series of standard solutions (minimum five concentrations covering working concentrations used for routine analysis) and analyze each solution minimum twice and record the instruments response. 
- Using the concentrations and corresponding instrument response, LOD and LOQ can be calculated as follows:

Let the linear regression equation be $\mathrm{Y}=\mathrm{a}+\mathrm{bX}$.

Where, $X$ and $Y$ are the variables (data of two parameters). Generally, $X$ is called the independent variable and $Y$, the dependent variable.

Take concentration on $\mathrm{X}$-axis and instrument response on $\mathrm{Y}$-axis.

"a" and " $b$ " are the regression constants. Further, "a" is known as the intercept and "b," the slope of the line.

Let $\left(X_{1}, Y_{1}\right),\left(X_{2}, Y_{2}\right),\left(X_{3}, Y_{3}\right) \ldots\left(X_{n}, Y_{n}\right)$ be the set of values required to be fit in the linear equation.

a. Method of arriving at " $a$ " and " $b$ " $\bar{y}$

i. Tabulate as given below:

\begin{tabular}{lc}
$X_{1}$ & $Y_{1}$ \\
$X_{2}$ & $Y_{2}$ \\
$\cdot$ & $\cdot$ \\
$\cdot$ & $\cdot$ \\
$X_{n}$ & $\cdot$ \\
\hline Mean, $=\bar{X}=\Sigma X / n$ & $\bar{Y}=\Sigma X / n$
\end{tabular}

ii. Calculate the following parameters:

$$
\begin{aligned}
& \Sigma x x=\Sigma(X-\bar{X})^{2}=\Sigma X^{2}-(\Sigma X)^{2} / \mathrm{n} \\
& \Sigma y y=\Sigma(Y-\bar{Y})^{2}=\Sigma Y^{2}-(\Sigma Y)^{2} / \mathrm{n} \\
& \Sigma x y=\Sigma X Y-(\Sigma X)(\Sigma Y) / \mathrm{n}
\end{aligned}
$$

iii. Calculate the slope " $b$, " and intercept "a" as given below:

$$
\begin{gathered}
b=\frac{\sum x y}{\sum x x} \\
a=\bar{Y}-b \bar{X}
\end{gathered}
$$

b. Method of calculation $\mathrm{r}$ (correlation coefficient) 


$$
r=\frac{\sum x y}{\sqrt{\sum x x \cdot \sum y y}}
$$

c. Method of calculation standard deviation for " $a$ " and " $b$ "

The standard deviation of the individual deviations of measured values in $Y$, above and below the linear line (fitted line) is:

$$
S y \cdot x=\sqrt{\frac{\left(\sum y y-\left\{\left(\sum x y\right)^{2} / \sum x x\right\}\right)}{n-2}}
$$

From this, the standard deviation for " $a$ " and " $b$ " are calculated.

Standard deviation

for " $\mathrm{a}$, " represented $=S y \cdot x \sqrt{\frac{\sum X^{2}}{n \sum x x}}$

as $S_{a}$

Standard deviation.

For " $\mathrm{b}$, " represented $=S y \cdot x \sqrt{\frac{1}{n \sum x x}}$

as $S_{b}$

\subsubsection{Application of $a, b$, and $S_{a}$ to obtain limit of detection and limit of quantitation}

When $S_{a}$ is obtained for a linear calibration line, then it provides a clear information on the standard deviation of the "Blank" (or Control) response from the instruments.

The LOD and LOQ can be worked out, as given below:

$$
\begin{aligned}
& \mathrm{LOD}=\frac{|\mathrm{a}|+3 S_{\mathrm{a}}}{\mathrm{b}} \\
& \mathrm{LOQ}=\frac{|\mathrm{a}|+10 S_{\mathrm{a}}}{\mathrm{b}}
\end{aligned}
$$

\section{Note:}

- The above calculations can be programmed in a computer but before every use, the computer program must be validated using the example given in section

- The above procedure can also be used for obtaining LOD and LOQ of the method from recovery test results by taking fortified concentration on $\mathrm{X}$-axis and obtained concentrations on Y-axis. 


\section{Example}

In this example, the linear regression equation is employed to find out the extent of linear response of an Detector to a reference analytical standard in the concentration range of about 0.2-3.0 ppm.

Each of these working standards is injected thrice ( $1 \mu \mathrm{l}$ per injection), and the peak area counts corresponding to the active ingredient peak are given below.

From the peak areas corresponding to each concentration level, the mean, standard deviation (SD) and coefficient of variation $(\% \mathrm{CV})$ are also calculated. The details were presented in Table 2.

Fitting the data of concentration of standard solution and mean detector response (peak area counts) in a linear equation

Let the equation be $Y=a+b X$.

Where, $Y=$ Mean peak area counts and $X=$ Concentration of standard solution, $\mu \mathrm{g} / \mathrm{ml}$.

The calculations were presented in Table 3.

\begin{tabular}{|c|c|c|c|c|c|c|}
\hline \multirow[t]{2}{*}{ Conc. of standard solution $(\mu \mathrm{g} / \mathrm{ml})$} & \multicolumn{3}{|c|}{ Peak area } & \multirow[t]{2}{*}{ Mean } & \multirow{2}{*}{$\begin{array}{l}\text { SD } \\
(n-1)\end{array}$} & \multirow[t]{2}{*}{$\% \mathrm{CV}$} \\
\hline & 1 & 2 & 3 & & & \\
\hline 0.1956 & 32,827 & 33,299 & 32,731 & 32,952 & 304 & 0.923 \\
\hline 0.4890 & 87,783 & 88,480 & 87,446 & 87,903 & 527 & 0.600 \\
\hline 0.9780 & 176,037 & 174,675 & 177,203 & 175,972 & 1265 & 0.719 \\
\hline 1.467 & 246,212 & 250,786 & 246,849 & 247,949 & 2477 & 0.999 \\
\hline 1.956 & 319,143 & 319,615 & 315,316 & 318,025 & 2358 & 0.741 \\
\hline 2.934 & 415,059 & 410,773 & 418,407 & 414,746 & 3827 & 0.923 \\
\hline
\end{tabular}

Table 2. Calculation details of mean, SD, and \%CV.

\begin{tabular}{lll}
\hline S1. no. & Y & X \\
\hline 1. & 32952 & 0.1956 \\
2. & 87903 & 0.4890 \\
3. & 175972 & 0.9780 \\
4. & 247949 & 1.4670 \\
5. & 318025 & 1.9560 \\
6. & 414746 & 2.9340 \\
\hline
\end{tabular}

Table 3. Calculation details of additional parameters. 


$$
\begin{array}{lcl}
\sum \mathrm{Y}=1277547 & \sum \mathrm{X}=8.0196 & \sum \mathrm{XY}=2424193.441 \\
\bar{Y}=212924.5 & \bar{X}=1.3366 & \mathrm{n}=6 \\
\sum \mathrm{Y}^{2}=3.7441177 \times 10^{11} & \sum \mathrm{X}^{2}=15.820245 &
\end{array}
$$

Using the above parameters, calculate the following

$$
\begin{aligned}
\sum x x & =\sum X^{2}-\left(\sum X\right)^{2} / \mathrm{n} \\
& =15.820245-(8.0196)^{2} / 6 \\
& =5.101248 \\
\sum y y & =\sum Y^{2}-\left(\sum Y\right)^{2} / \mathrm{n} \\
= & 3.7441176 \times 10^{11}-(1277547)^{2} / 6 \\
= & 1.0239070 \times 10^{11} \\
= & \sum X Y-\left(\sum X\right)\left(\sum Y\right) / n \\
= & 2424193.441-(1277547)(8.0196) / 6 \\
= & 716624.12
\end{aligned}
$$

Calculation of $a, b$, and $r$

$$
\begin{aligned}
& \mathrm{b}=\frac{\sum x \mathrm{x}}{\sum \mathrm{xx}} \\
&= \frac{716624.12}{5.101248} \\
&= 140480.16 \\
& \mathrm{~b}=\frac{\sum \mathrm{xy}}{\sum \mathrm{xx}} \\
&=\frac{716624.12}{5.101248} \\
&=140480.16 \\
& \mathrm{a}=\overline{\mathrm{Y}}-\mathrm{b} \overline{\mathrm{X}} 212924.5-140480.16 \times 1.3366 \\
&=25158.718 \\
& r=\frac{\sum x y}{\sqrt{\sum x x \cdot \sum y y}} \\
& r=\frac{716624.12}{\sqrt{1.0239070 X 10^{11} \mathrm{X} 5.101248}}=0.99157
\end{aligned}
$$

Note: Sometimes $r^{2}$ is also used to express the goodness of fit.

Calculation of standard deviation for $a$ and $b$ : 


$$
\begin{aligned}
\text { Sy.x } & =\sqrt{\frac{\sum y y-\left\{\left(\sum x y\right)^{2} / \sum x x\right\}}{n-2}} \\
& =\sqrt{\frac{\left(1.0239070 \times 10^{11}\right)-\left\{(716624.12)^{2} /(5.101248)\right\}}{6-2}} \\
& =20731.806
\end{aligned}
$$

The standard deviation for a is calculated as:

$$
\begin{aligned}
S_{a} & =S y \cdot x \sqrt{\frac{\sum X^{2}}{n \sum x x}} \\
& =20731.806 \sqrt{\frac{15.820245}{6 \times 5.101248}} \\
& =14905
\end{aligned}
$$

The standard deviation for $b$ is calculated as

$$
\begin{aligned}
S_{b} & =S y \cdot x \sqrt{\frac{1}{n \cdot \sum x x}} \\
& =20731.806 \sqrt{\frac{1}{6 \times 5.101248}}
\end{aligned}
$$

Note: Assay procedures vary from highly exacting analytical determinations to subjective evaluations of attributes. Therefore different test methods require different validation schemes.

\section{Category I}

Analytical methods for quantitation of major excipients and/or active ingredients, and preservatives in finished goods.

\section{Category II}

Analytical methods for determination of impurities or degradation compounds in finished goods. These methods include quantitative assays and limit tests, titrimetric and bacterial endotoxin tests.

\section{Category III}

Analytical methods for determination of performance characteristics, e.g., sterility testing, dissolution and drug release for pharmaceutical products.

\section{Data Elements Required for Assay Validation.}

Details of required validation parameters of assay presented in Table 4. 


\begin{tabular}{|c|c|c|c|c|}
\hline Analytical parameters & $\begin{array}{l}\text { Assay } \\
\text { category } 1\end{array}$ & $\begin{array}{l}\text { Assay category } 2 \\
\text { quantitative }\end{array}$ & Limit Test & $\begin{array}{l}\text { Assay } \\
\text { category III }\end{array}$ \\
\hline Assay accuracy & Yes & Yes & * & * \\
\hline Precision & Yes & Yes & No & Yes \\
\hline Specificity & Yes & Yes & Yes & * \\
\hline Limit of detection & Yes & Yes & Yes & * \\
\hline Limit of quantitation & Yes & Yes & No & * \\
\hline Linearity & Yes & Yes & No & * \\
\hline Range & Yes & Yes & * & * \\
\hline Robustness & * & * & * & * \\
\hline
\end{tabular}

*May be required depending on the specific test.

Table 4. Validation parameters of assay [16].

\section{Conclusions}

Analytical validation data playing a fundamental role in pharmaceutical industry, pesticide industry for releasing the economic batch and long term stability information consequently, the records must be produced to suited regulatory authority requirements.

\section{Author details}

Tentu Nageswara Rao

Address all correspondence to: tentu6581@rediffmail.com

Department of Chemistry, Krishna University, Machilipatnam, Andhra Pradesh, India

\section{References}

[1] Validation of analytical procedure: Methodology Q2B. In: ICH Harmonized Tripartite Guidelines. Geneva, Switzerland. 1996. pp. 1-8

[2] CIPAC Document No. 3807-Guidelines on method Validation to be performed in support of analytical methods for agrochemical Formulations, cipac.org on 28 July 2003

[3] International Standard ISO 5725. 1986. Precision of test methods-Repeatability and reproducibility

[4] ISO standard 11095. 1996. Linear calibration using reference material 
[5] Horwitz W. Evaluation of analytical methods used for regulation of foods and drugs. Analytical Chemistry. 1982;54(1):67A-76A. DOI: 10.1021/AC00238A765

[6] Thompson M, Ellison SLR, Wood R. Harmonised guidelines for single laboratory validation of method of analysis. Pure and Applied Chemistry. 2008;74(5):835-855

[7] European Commission. Annex 15. EU guide to good manufacturing practice: Qualification and validation. 2010;4:1-10

[8] Ravichandran V, Shalini S, Sundram KM, Rajak H. Validation of analytical methodsStrategies \& importance. International Journal of Pharmacy and Pharmaceutical Sciences. 2010;2(3):340-345

[9] Tangri P, Rawat PS, Jakhmola V. Validation: A critical parameter for quality control of pharmaceuticals. Journal of Drug Delivery \& Therapeutics. 2012;2(3):34-40

[10] Sharma A, Sharma R. Validation of analytical procedures: A comparison of ICH Vs Pharmacopoiea (USP) Vs FDA. International Research Journal of Pharmacy. 2012;3(6):39-42

[11] Lambert J. Validation guidelines for pharmaceutical dosage forms. Health Canada Health Products and Food Branch Inspectorate. 2004;26:7-15

[12] Prabh SS, Gagan S. Analytical method development and validation. Journal of Pharmacy Research. 2011;4(7):2330-2332

[13] Ramamurthy M, Sarvanakumar K. Pharmaceutical validation. The Eastern Pharmacist. 1997;476:45-47

[14] Agalloco J. Validation: An unconventional review and reinvention. PDA. J. Pharm Sci Tech. 1995;49:175-179

[15] INRA Quality Policy and Quality Guidelines for the Research and Experimental Units. 2013

[16] Haider I. Section VAL 1100.00. In: Validation Standard Operating Procedures. A Step by Step Guide for Achieving Compliance in the Pharmaceutical Medical Device and BiotechIndustries. Boca Raton: CRC Press LLC; 2001 
